\title{
Spectrophotometric redshifts
}

\section{A new approach to the reduction of noisy spectra and its application to GRB 090423}

\author{
M. Stefanon ${ }^{1}$, A. Fernandez-Soto ${ }^{2}$, and D. Fugazza ${ }^{3}$ \\ 1 Observatori Astronomic de la Universitat de Valencia, c/ Catedratico Agustin Escardino Benlloch 7, Paterna, 46980 Valencia, Spain \\ e-mail: mauro.stefanon@uv.es \\ 2 Instituto de Fisica de Cantabria (CSIC-UC), Edificio Juan Jorda, Av. los Castros s/n, 39005 Santander, Spain \\ e-mail: fsoto@ifca.unican.es \\ 3 INAF - Osservatorio Astronomico di Brera, via Bianchi 46, 23807 Merate(LC), Italy \\ e-mail: dino.fugazza@brera.inaf.it
}

Received 13 May 2010 / Accepted 27 September 2010

\begin{abstract}
Context. The measurement of redshifts for objects on the verge of instrumental observability is difficult and prone to error. This is especially true for almost featureless spectra, as is the case for GRB afterglows. They can be detected out to the farthest distances, and usually spectroscopy poses a serious problem because they fade quickly.

Aims. We have developed a new method that is close in philosophy to the photometric redshift technique, which can be applied to spectral data with a very low signal-to-noise ratio. We intend to measure redshifts, while minimising the dangers posed by the usual extraction techniques.

Methods. GRB afterglows have generally very simple optical spectra, which can be described well by a pure power law, over which the separate effects of absorption and reddening in the GRB host, the intergalactic medium, and our own Galaxy are superimposed. We model all these effects over a series of template afterglow spectra to produce a set of clean spectra that reproduce what would reach our telescope. We also carefully model the effects of the telescope-spectrograph combination and the properties of noise in the data, which are then applied to the template spectra. The final templates are compared to the two-dimensional spectral data, and the basic parameters (redshift, spectral index, hydrogen absorption column) are estimated with statistical tools.

Results. We show how our method works by applying it to our data of the NIR afterglow of Swift GRB 090423. At $z \approx 8.2$, this was the most distant object ever observed. Our team took a spectrum using the Telescopio Nazionale Galileo, which we use in this article to derive its redshift and its intrinsic neutral hydrogen column density. Our best fit yields $z=8.4_{-0.03}^{+0.05}$ and $N(\mathrm{HI})<5 \times 10^{20} \mathrm{~cm}^{-2}$, but with a highly non-Gaussian uncertainty including the redshift range $z \in[6.7,8.5]$ at the 2-sigma confidence level.

Conclusions. Our method will be useful for maximising the recovered information from low-quality spectra, particularly when the set of possible spectra is limited or easily parameterisable (as is the case in GRB afterglows), while at the same time ensuring adequate confidence analysis.
\end{abstract}

Key words. techniques: spectroscopic - cosmology: observations - gamma-ray burst: general

\section{Introduction}

Measuring redshifts is one of the most important techniques in astronomy; however, it is also one that depends critically on the quality of the available data. Because of its nature it is sometimes difficult to attest to the quality of the results, since no quantitative error estimates are obtained. In the optical range, the standard approach is to reduce all the available data to a one-dimensional array, which is flux- and wavelength-calibrated with the help of auxiliary data. In most cases, detection of emission and/or absorption lines is necessary for a valid measurement, although in some instances, only the continuum and some basic spectral features (e.g. breaks) are needed. Even the latter is sometimes difficult because of the paucity of photons. Ideally, in cases of low signal-to-noise data, one would bin the spectrum in the wavelength direction, but even this is sometimes useless. Moreover, information is often lost in the process of extracting the spectrum.
A different approach from the informational point of view would be to choose a model that represents the best possible fit to the available two-dimensional spectral data. This is actually the approach used by photometric redshift techniques, when a series of spectral energy distributions are considered at different redshifts and converted into photometric data that can be compared with the available photometry. It is also the method that has become standard in high-energy ( $\mathrm{X}$ and gamma-ray) spectroscopy, where the models are convolved with the instrumental response and compared to the data, instead of the data being extracted and calibrated. In order for this kind of approach to work, at least three conditions need to be fulfilled:

- the real spectrum must be included in the family of models under analysis. This would be relatively difficult, for instance, in the case of quasars or galaxies at moderate resolution, because the intrinsic scatter amongst different types or models is very large. However, GRB afterglows make for an excellent example, as their intrinsic optical spectrum can 


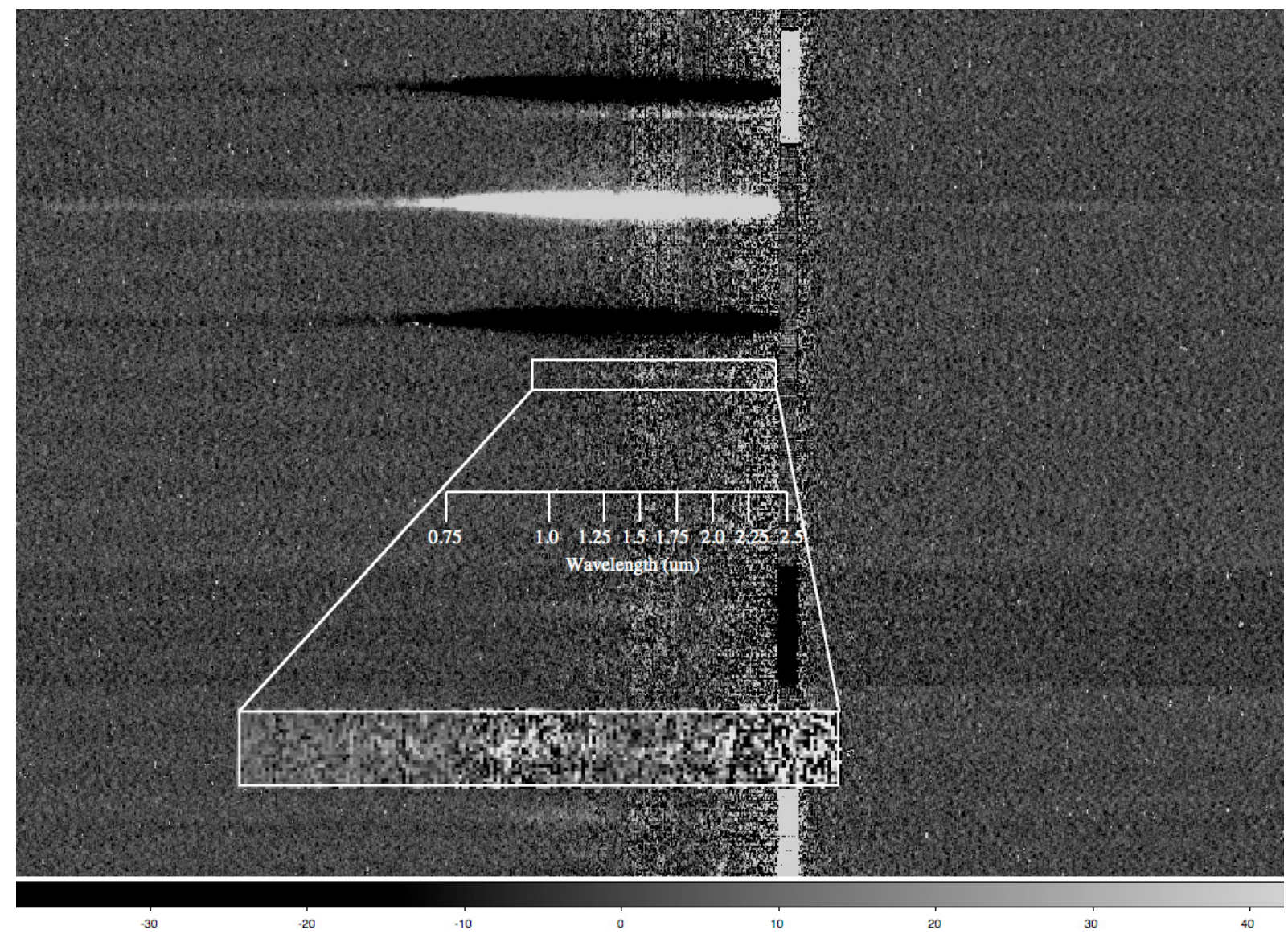

Fig. 1. The combined two-dimensional spectrum used as input data. The white rectangle identifies the region of the GRB afterglow spectrum that was used for the analysis. The box height is equal to $4 \times f w h m$, corresponding to 21 pixels which ensures that we completely include both wings of the Gaussian. The inset shows a magnification of the same region.

usually be approximated very well with a single power law, where the effects of the GRB host gas and dust, the intergalactic medium, and our local extinction can be superimposed;

- the technical characteristics of the instrument must be well known, in order to model their effect into the simulated data. This includes the total wavelength-dependent efficiency, any possible geometric distortions in the spectral direction, and of course the exact position of the target in the slit image;

- the characteristics of the noise in the CCD must also be modelled accurately, so that the statistical analysis will accurately estimate the model parameters and also the uncertainties associated to them.

Even though we have not mentioned it explicitly, it is of course necessary to ensure an accurate first reduction of the data, going from the raw individual images to the combined twodimensional spectrum, which constitutes the input data for our analysis.

\section{Description of the data}

To describe our work in detail, we concentrate on the particular case for which we developed our original idea, thus we start by describing those data.

\subsection{GRB 090423 afterglow data}

GRB 090423 was a gamma-ray burst detected by the Swift satellite on April 23, 2009 (Krimm et al. 2009). Early observations in the optical and near infrared distinctly pointed towards the possibility of it being a high-redshift object, when it went undetected for all observers using visible bands, but showed as a relatively bright near-infrared source (Tanvir et al. 2009a; Cucchiara et al. 2009a). Photometric data alone indicated a very highredshift nature, with basically zero dust absorption (Cucchiara et al. 2009b; Olivares et al. 2009). Our group used the Italian $3.6 \mathrm{~m}$ Telescopio Nazionale Galileo on the island of La Palma, to obtain a low-resolution spectrum using the Amici prism with the spectrograph NICS (Oliva 2003), and measured its redshift to be $z=8.1_{-0.3}^{+0.1}$ (Thoene et al. 2009; Fernandez-Soto et al. 2009; Salvaterra et al. 2009). A compatible result $\left(z=8.23_{-0.07}^{+0.06}\right)$ was reached independently by Tanvir et al. (2009b,c) using two sets of higher-quality data obtained with the VLT in Chile.

The Amici spectrum covers in a single exposure the wavelength range $0.8-2.5 \mu \mathrm{m}$ with very low resolution $(R \approx 50)$ but very high efficiency, and thus became the ideal choice for this kind of analysis. We obtained a total of $128 \mathrm{~min}$ of on-target exposure time. The exposures were dithered following the usual NIR technique, and combined into a single two-dimensional frame, which is showed in Fig. 1. The slit was positioned with the help of a nearby star, whose extracted spectrum will be one of the keys in our analysis.

The position of the star along the slit (measured at the reference position $X=600$ in the CCD frame) is $Y=765$. The 
angular distance between the reference star and the afterglow was $\approx 30$ arcsec, which corresponds to 120 pixels along the slit. To avoid possible issues caused by misalignments or the effect of distortions in the focal plane, we use this distance only as a reference, and perform a careful recentring, as described in the next section.

\section{Description of the method}

Our aim is to reproduce the spectrum of the afterglow of GRB 090423 as perfectly as possible, and to reconstruct the spectral equivalent of the wavelength-dependent point spread function as generated when the light passes through the atmosphere, telescope, and instrument optics, and reaches the detector. We present in this section the different steps to reach this objective.

\subsection{Model spectra}

We have created a library of model spectra, where there are three basic input parameters: the redshift $z$, the slope $\alpha$ in the powerlaw spectral model $f_{v} \propto \lambda^{\alpha}$, and the total neutral hydrogen column density in the host interstellar medium N(HI), which produces a strong damped Lyman alpha (DLA) profile at the host redshift. It is important to include this profile in the analysis, because a dense $\left(N(\mathrm{HI}) \gtrsim 10^{21} \mathrm{~cm}^{-2}\right.$ ) DLA profile would displace the position of the break, thus mimicking a higher redshift. A fourth parameter, the apparent magnitude normalisation in the observed $K$ band (at the epoch of our observations) $m_{K}$, will be left as an uninteresting parameter, and it is directly fitted to the data during the process.

The effect of the Inter-Galactic Medium (IGM) at the redshifts of interest $(z \gtrsim 6)$ is very simple to include. At such a high redshift, the HI absorption is complete - within our observational capabilities - below the Lyman- $\alpha$ line, and as such we include it in the models (Yoshii \& Peterson 1994). The putative effects of a significatively different neutral fraction in the IGM were deliberately neglected for two reasons: on one hand, the literature on GRB 090423 points to a normal environment (i.e. neutral, see Tanvir et al. 2009); on the other, some authors have shown that those effects are difficult to model and are challenging to observe even with better quality data (e.g. Patel et al. 2010).

In other cases one would of course need different parameters. It could be necessary to add dust extinction either at the host or by the Milky Way (or both), with the amount of extinction and even its grain type left as free parameters. We do not consider it here, because the available afterglow photometry indicates a blue object with an almost complete lack of intrinsic extinction (Fernandez-Soto et al. 2009; and Tanvir et al. 2009c, in particular their Fig. 2 which shows how the spectral slope is represented by a pure power law). Moreover, given the narrow rest-frame wavelength range that we are observing $(\lambda \approx 1200-2200 \AA)$, as well as the resolution and signal-to-noise ratio of our data, there is an almost perfect degeneracy between the amount of dust extinction and a change in spectral slope ${ }^{1}$.

Figure 2 shows a selection of spectral templates that sample part of the parameter space. The full range covered by our templates is $\alpha \in[-1,3], z \in[5,10]$, and $N(\mathrm{HI}) \in\left[10^{20}, 10^{24}\right] \mathrm{cm}^{-2}$. All three ranges safely include the expected values of each variable. All models are normalised to have $A B_{K}=21.3$, a value

\footnotetext{
${ }^{1}$ We used the Schlegel et al. (1998) maps to include in the templates the effect of Milky Way dust at the level of $E(B-V)=0.03$.
}

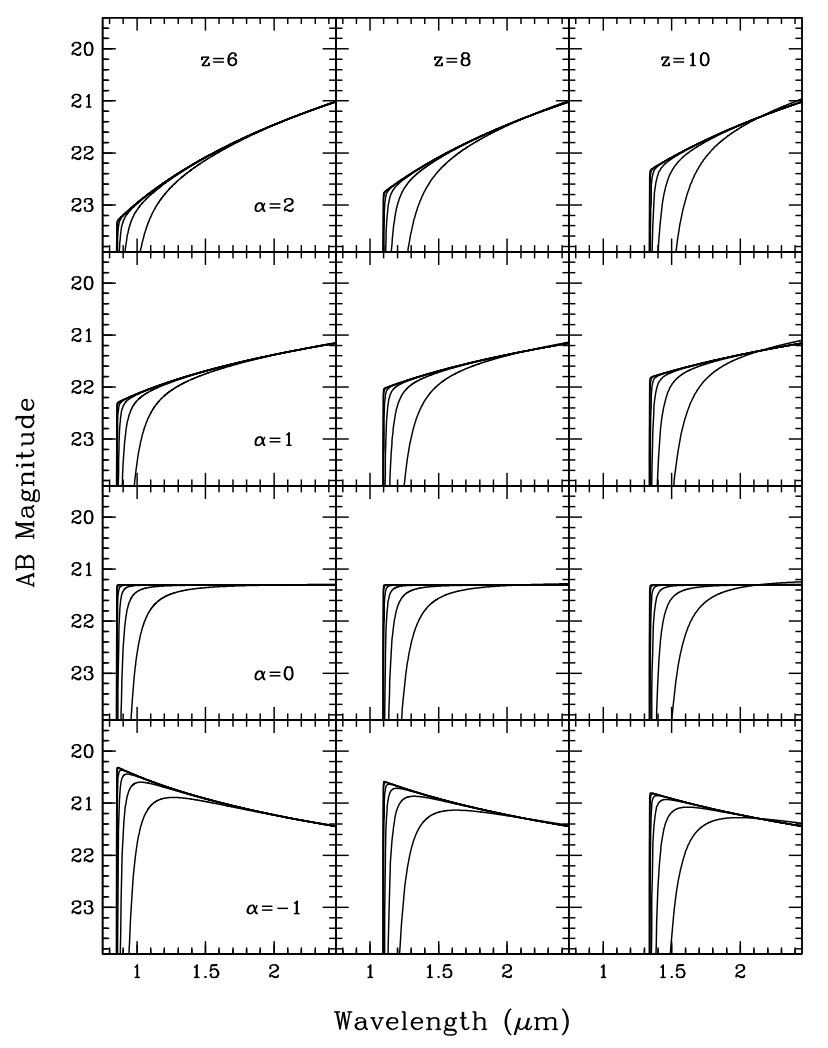

Fig. 2. Some of the templates used for the analysis. Each column corresponds to a different redshift as labelled in the top panels, and each line to a different spectral slope, as labelled on the leftmost column. Within each panel, the different curves correspond to values of $\log [N(\mathrm{HI})]$ varying from 19 to 24 in steps of one.

measured by GROND (Tanvir et al. 2009c) at almost exactly the same time our observations were performed. It must be pointed out, however, that, because of the possibility of slit losses affecting the detected flux, we leave the normalisation factor for the flux as a free parameter, as explained in the next section.

\section{2. $C C D$ and instrumental characteristics}

Once satisfied with the set of spectral templates, we need to characterise the observations in terms of spectral resolution, efficiency of the instrument at different wavelengths, noise characterics of the detector, and position of the spectrum along both the spectral and spatial directions.

\subsubsection{Instrumental characteristics}

We used an archival solution to calibrate the Amici spectrum in wavelength. As described in Fernandez-Soto et al. (2009) we needed to add an offset of 5 pixels, determined via comparison with the observed sky absorption features.

As we mentioned in the previous section, there is a nearby star that falls within the slit - it was in fact used to position the slit, as the afterglow was too dim to be pointed at directly. Its spectral type is M4V, as determined via available SDSS and 2MASS photometry (Adelman-McCarthy et al. 2008; Skrutskie et al. 2006, see Table 1 for the complete data). We used the corresponding spectrum from the Bruzual-Persson-Gunn-Stryker library (Bruzual et al. 1996) to determine the total efficiency of the instrument as a function of wavelength. In the $0.95-1.1 \mu \mathrm{m}$ region, corresponding to the Lyman break of a $z \approx 8$ source - the 
Table 1. Stellar data.

\begin{tabular}{lc}
\hline \hline Parameter & Value \\
\hline SDSS name & J095535.28+180903.8 \\
RA (J2000) & $09: 55: 35.286$ \\
Dec (J2000) & $+18: 09: 03.88$ \\
Spectral Type & M4V \\
$u_{\text {SDSS }}$ & $22.17 \pm 0.21$ \\
$g_{\text {SDSS }}$ & $19.27 \pm 0.01$ \\
$r_{\text {SDSS }}$ & $17.78 \pm 0.01$ \\
$i_{\text {SDSS }}$ & $16.54 \pm 0.01$ \\
$z_{\text {SDSS }}$ & $15.91 \pm 0.01$ \\
$J_{2 \text { MASS }}$ & $14.52 \pm 0.03$ \\
$H_{2 \text { MASS }}$ & $13.97 \pm 0.04$ \\
$K_{2 \text { MASS }}$ & $13.77 \pm 0.05$ \\
\hline
\end{tabular}

Notes. SDSS and 2MASS photometry taken from their respective catalogues, expressed in their usual reference $-A B$ magnitudes for SDSS, Vega-based for 2MASS.

most prominent feature in our GRB spectrum - the spectrum of an M4V star is free of strong spectral features. This, considered together with the low resolution of the spectra and the fact that the M4V star shows even less features at higher wavelengths, allows us to rely on the total instrumental efficiency we derived.

It must be noted that there is a free factor involved in the calculations, as we cannot ensure that the slit losses in the stellar spectrum are the same in the one corresponding to the afterglow. However, as long as the pointing was reasonably accurate - and we can assume it was from the comparison of the fluxes - at least to first order, it will be a single number (i.e., not wavelengthdependent) since the slit angle is obviously the same for both objects.

The seeing at the time of the observations was $\approx 1.4$ arcsec in the $J$ band, as measured in the acquisition images. The slit width was 1.0 arcsec, so we expect some slit losses, and no degradation in resolution induced by the slit width. We show in Fig. 3 the measured total efficiency, obtained as the (arbitrarily normalised) ratio of the counts to the model spectrum of the star.

As it is usually done when reducing spectroscopic data of very faint sources, we assume that the shape of the afterglow spectrum in the CCD follows the trace left by the much brighter star (plotted in Fig. 3, second panel from the top), which can easily be traced at all wavelengths from 0.8 to 2.5 microns. We determined the distortions along the dispersion axis of the stellar spectrum, fitted it via Legendre polynomials, and used it to define the template spectrum position. We determined the vertical offset by using a zero-order solution (an $f_{v}$-flat spectrum at $z=8.4$ with $\mathrm{HI}$ absorption of $10^{20} \mathrm{~cm}^{-2}$ ) and displacing it vertically, evaluating its likelihood at different positions when compared to the CCD data. We measured an offset of 115.4 pixels (equivalent to 28.8 arcsec).

The FWHM in the spatial direction of the stellar spectrum varies with wavelength from $\approx 1.5 \operatorname{arcsec}$ at $2.2 \mu \mathrm{m}$ to $\approx 1.2 \operatorname{arcsec}$ at $\approx 0.8 \mu \mathrm{m}$ (corresponding to $\approx 5.8$ to $\approx 4.9$ pixels on our plate scale). We used a smoothed fit to those values (also shown in Fig. 3) to reproduce the afterglow spectrum.

\subsubsection{CCD characteristics}

The CCD image we are working with is the result of a careful reduction procedure following the usual steps for NIR spectroscopy. We did, however, perform one extra check: we measured the background in detail, to ensure that it is flat in both the spectral and spatial directions, and found no trend in any of them, with the background flat to a fraction of the CCD noise.

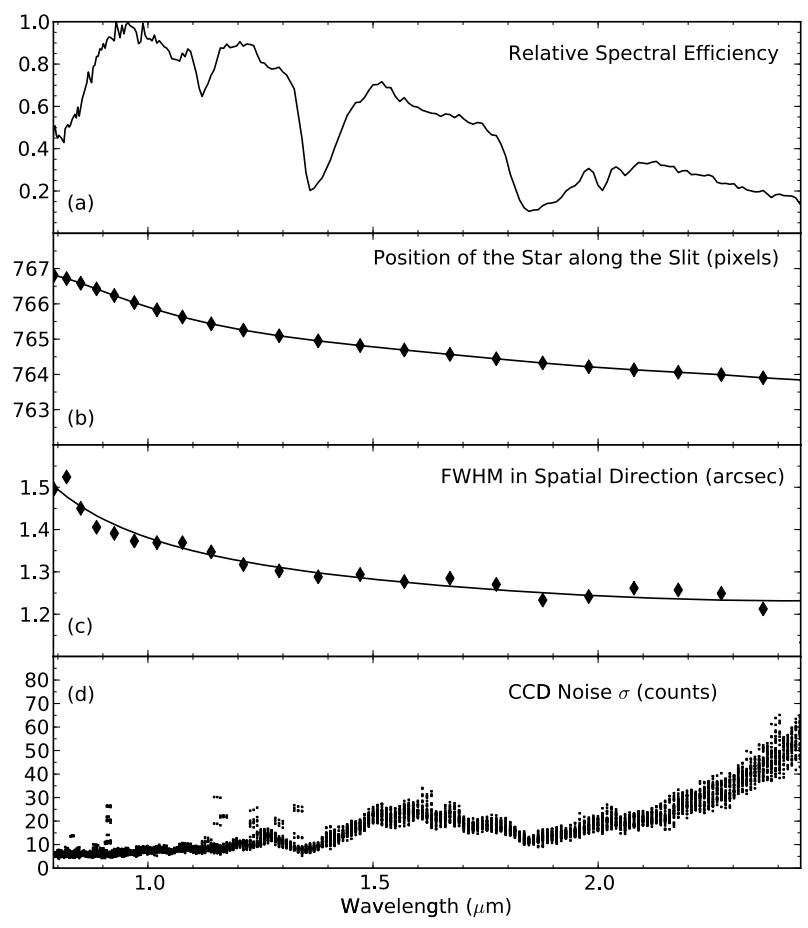

Fig. 3. Total efficiency of the atmosphere, telescope, and instrument combination a). Changes in the spectral position b) and width c) with wavelength, and projection of the measured CCD noise (rms counts per pixel) as a function of wavelength $\mathbf{d}$ ).

We also estimated the CCD noise using different methods. This is a very important step, because we want not only to determine the basic parameters of the GRB afterglow (redshift, spectral slope, and neutral hydrogen column density), but also to measure confidence limits on both. We decided that the best method consists in using 7 rectangular areas of equal size in the CCD, each one covering the interval $X \in[480,650]$ and 21 pixels in the $Y$ direction. Those boxes were chosen in areas that are free of any (positive or negative) feature. With them we estimated the noise as a function of $X$ position (that is, wavelength) by using a $3 \times 3$ grid around each pixel and all 7 independent images. In this way we obtained a $170 \times 21$ mini-CCD noise frame, which we will use for the subsequent chi-square analysis. The projection of this noise array on the $Y$ direction is also shown at the bottom in Fig. 3.

We finally computed the noise expected from an ideal $\chi^{2} \equiv 1$ relation when comparing each one of the noise regions to an empty (zero counts) one. The average of the obtained noise values differs from the RMS of our noise array by $\approx 0.3 \%$.

\subsection{Application of the method}

We used all the knowledge about the instrument gathered in the previous subsection to create replicas of the GRB afterglow spectrum, for each one of the templates described in Sect. 3.1.

In brief, we choose a spectrum template combination (i.e., values of $z, \alpha$, and $N(\mathrm{HI})$ ) and generate a one-dimensional spectrum using them. We redden this spectrum using the measured Galactic value $E(B-V)=0.03$. Then this spectrum is converted to a two-dimensional image, using the measured efficiency, adjusting and block-averaging the wavelength axis to the known dispersion solution of the Amici prism, and convolving it in the spectral direction with a Gaussian function of the measured FWHM at each wavelength. This two-dimensional 

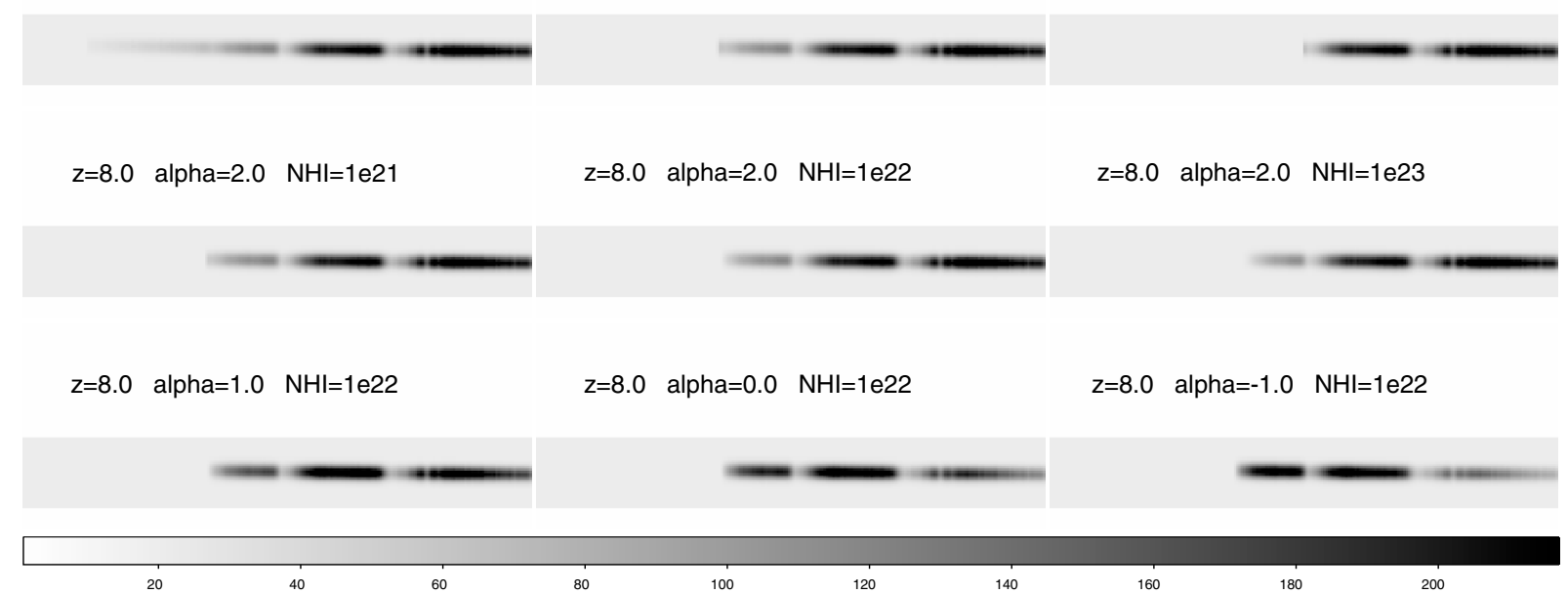

Fig. 4. Some of the templates that were shown in Fig. 1 are shown here as two-dimensional arrays, after applying the procedures described in Sect. 3.3.

spectrum is forced to follow the trace that was measured with the stellar spectrum, and is set on a mini-CCD matrix measuring $170 \times 21$ pixels, as was described above for the noise image. Figure 4 shows some of the same spectra that were presented in Fig. 2, now converted to two-dimensional images with this process.

Each of them is then compared to the real data, using the section of the CCD that contains the GRB afterglow spectrum, centred to the same position of each of the template frames. We do this comparison using a $\chi^{2}$ fit, where the noise array corresponds to the one that was obtained in the previous section. Calling the template $\mathcal{T}(z, \alpha, N(\mathrm{HI}))$, the CCD data $\mathcal{D}$, and the noise matrix $\mathcal{S}$, and remembering each one of them represents a $170 \times 21$ matrix, one obtains

$\chi^{2}[z, \alpha, N(\mathrm{HI})]=\sum_{i=1}^{170} \sum_{j=1}^{21} \frac{\left[A \mathcal{T}(z, \alpha, N(\mathrm{HI}))_{i j}-\mathcal{D}_{i j}\right]^{2}}{\mathcal{S}_{i j}^{2}}$

where $A$ represents a normalisation parameter for the flux. This is actually fixed for each template by minimising $\chi^{2}$, which renders

$A=\frac{\sum_{i=1}^{170} \sum_{j=1}^{21} \mathcal{D}_{i j} \mathcal{T}(z, \alpha, N(\mathrm{HI}))_{i j} / \mathcal{S}_{i j}^{2}}{\sum_{i=1}^{170} \sum_{j=1}^{21} \mathcal{T}(z, \alpha, N(\mathrm{HI}))_{i j}^{2} / \mathcal{S}_{i j}^{2}}$.

Thus, once the normalisation $A$ is fixed, the calculation of $\chi^{2}$ is straightforward for each template.

\section{Results}

In Fig. 5 we present the result of the calculation above, projected on the different planes of parameter space. Throughout our analysis, we associated the $68.3 \%$ confidence level to $1 \sigma$ and $95.4 \%$ to $2 \sigma$. As can be seen, the best fit corresponds to $z=8.40, \alpha=1.2, N(\mathrm{HI})=10^{20.0} \mathrm{~cm}^{-2}$. We calculated the confidence regions corresponding to the best fit on each of the individual parameters, reaching the results presented in Table 2. We must point out that the best-fit solution represents a value of $\chi^{2}=4019$. This value indicates a good fit for our problem, which has $\approx 3600$ degrees of freedom, albeit obviously most of them void in terms of information content.

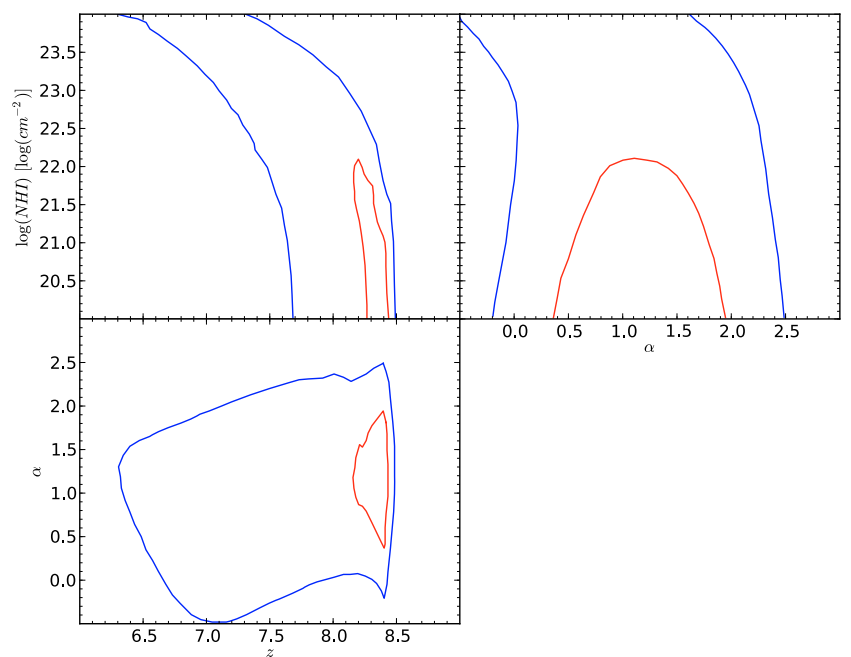

Fig. 5. Results of the fitting procedure, projected on each of the twodimensional planes of parameter space. Red (blue) contours correspond to $1 \sigma(2 \sigma)$ confidence levels.

Table 2. Results of the fitting procedure.

\begin{tabular}{lccc}
\hline \hline Parameter & Value & $1 \sigma$ & $2 \sigma$ \\
\hline Redshift & 8.40 & $(8.38,8.45)$ & $(6.67,8.49)$ \\
Spectral slope & 1.2 & $(0.7,1.8)$ & $(0.1,2.2)$ \\
$\log [N(\mathrm{HI})]$ & 20.0 & $(<20.7)$ & - \\
\hline
\end{tabular}

As can be observed, there is no lower limit to the neutral hydrogen column density. This is a natural observational consequence of the fact that below $N(\mathrm{HI}) \approx 2 \times 10^{20} \mathrm{~cm}^{-2}$ there is no damped profile, and the absorption is not significant at our resolution and signal-to-noise level. On the other end of the column density scale, we stop our analysis at $N(\mathrm{HI})=10^{24} \mathrm{~cm}^{-2}$, a value high enough to include even the densest absorbers ever observed. At $2 \sigma$ level, even higher values could be accomodated, paired to lower redshifts $z<6.5$ and flatter spectral slopes $\alpha<0.5$.

Figure 6 shows the best-fit spectrum, seen in the two top panels both as a one-dimensional plot and as a clean 


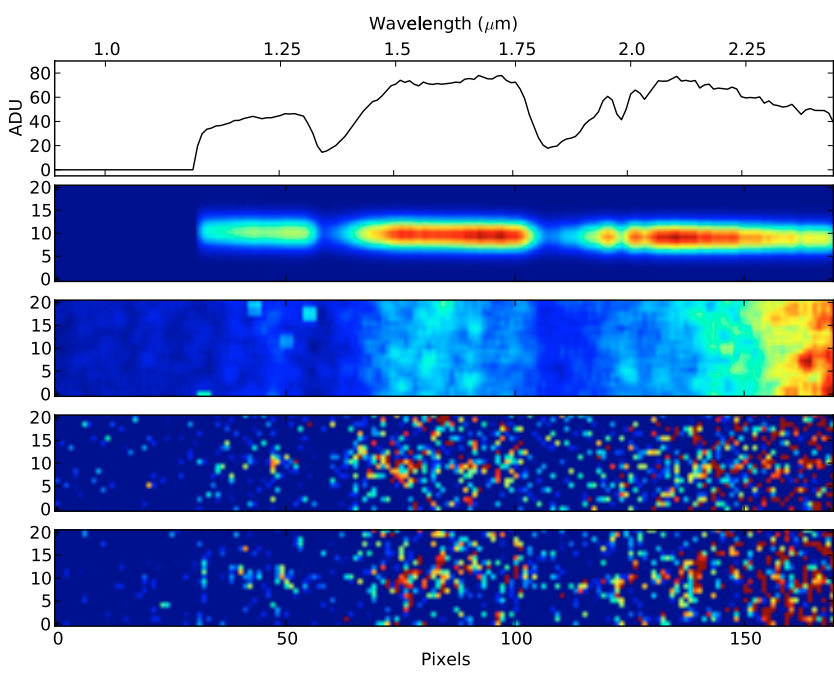

Fig. 6. The best-fit spectrum, convolved with the instrument response. It is shown in the top panel as a one-dimensional spectrum and as a clean two-dimensional array in the second one. The third panel shows a $1 \sigma$ noise two-dimensional array in the CCD area corresponding to the afterglow spectrum, as described in Sect. 3.2.2. The fourth panel shows one realisation of the CCD noise plus the best-fit spectrum, which can be directly compared to the real data at the bottom panel. The contrast scale is the same in the two last panels, but not in the previous ones.

two-dimensional model, and compared to the real data in the two lower panels.

We may also use the spectral slope of the GRB afterglow spectrum, as measured from broad-band NIR photometry, as a prior condition in our analysis. In this way, using the 1-sigma limit presented in Salvaterra et al. (2009) for the spectral slope, one would obtain a more stringent limit on the redshift, approximately $z>7.5$ at the 2-sigma confidence level.

\section{Conclusions}

We have presented a different method for the analysis of spectroscopic data, akin in spirit to the one used in X-ray astronomy and also in photometric resdhift techniques. This method does not attempt to extract a one-dimensional spectrum from the CCD data. On the other hand, we generate simulated two-dimensional spectra from template spectra of known characteristics, and decide which one fits the real data best, choosing the best-fit parameters as the solutions to the problem.

We used this method to carefully analyse a very low signal-to-noise spectrum of the afterglow of the very distant GRB 090423, showing that it is possible to extract information from this kind of data, more than is usually assumed. Our best result $\left(z=8.40_{-0.02}^{+0.05}\right)$ is slightly higher than previous results using the same data (Salvaterra et al. 2009) or data from other sources (Tanvir et al. 2009c), although still compatible well within the $2 \sigma$ confidence level. We consider our confidence intervals to be reliable, given the full analysis of the noise characteristics performed in this work.
The same method can be applied to other cases where the source belongs to a well-defined spectral class of objects, and there is a need to extract the maximum possible information from low-quality data. This is usually the case for faint, rapidly fading GRB afterglows, and we expect this method to be used in future measurements.

Acknowledgements. The authors gratefully acknowledge the support and effort of all members in the CIBO collaboration (Consorzio Italiano Burst Ottici), particularly Elisabetta Maiorano as PI of the proposal whence the Telescopio Nazionale Galileo GRB 090423 data were obtained, and Stefano Covino for his help with the manuscript.

A.F.S. acknowledges support from the Spanish MICYNN projects AYA200614056 and Consolider-Ingenio 2007-32022, and from the Generalitat Valenciana project Prometeo 2008/132. It is also a pleaure to acknowledge the support of the Observatori Astronomic de la Universitat de Valencia during the development of this work.

This work is partly based on observations collected at the TNG. The TNG telescope is operated on the island of La Palma by the Centro Galileo Galilei of the INAF in the Spanish Observatorio del Roque de Los Muchachos of the Instituto de Astrofísica de Canarias.

This work made use of public data from the SDSS and 2MASS surveys. The Two Micron All Sky Survey is a joint project of the University of Massachusetts and the Infrared Processing and Analysis Center/California Institute of Technology, funded by the National Aeronautics and Space Administration and the National Science Foundation. Funding for the SDSS and SDSS-II has been provided by the Alfred P. Sloan Foundation, the Participating Institutions, the National Science Foundation, the US Department of Energy, the National Aeronautics and Space Administration, the Japanese Monbukagakusho, the Max Planck Society, and the Higher Education Funding Council for England. The SDSS Web Site is http://www.sdss.org/. The SDSS is managed by the Astrophysical Research Consortium for the Participating Institutions. The Participating Institutions are the American Museum of Natural History, Astrophysical Institute Potsdam, University of Basel, University of Cambridge, Case Western Reserve University, University of Chicago, Drexel University, Fermilab, the Institute for Advanced Study, the Japan Participation Group, Johns Hopkins University, the Joint Institute for Nuclear Astrophysics, the Kavli Institute for Particle Astrophysics and Cosmology, the Korean Scientist Group, the Chinese Academy of Sciences (LAMOST), Los Alamos National Laboratory, the Max-PlanckInstitute for Astronomy (MPIA), the Max-Planck-Institute for Astrophysics (MPA), New Mexico State University, Ohio State University, University of Pittsburgh, University of Portsmouth, Princeton University, the United States Naval Observatory, and the University of Washington.

\section{References}

Adelman-McCarthy, J. K., Agüeros, M. A., Allam, S. S., et al., 2008, ApJS, 175, 297

Bruzual, G., Persson, E., Gunn, J., \& Stryker, L. 1996, BruzualPersson-Gunn-Stryker Spectrophotometry Atlas, http://www.stsci.edu/ instruments/observatory/cdbs/astronomical_catalogs.html Cucchiara, A., Fox, D. B., \& Berger, E. 2009a, GCN Circ., 9209

Cucchiara, A., Fox, D. B., \& Berger, E. 2009b, GCN Circ., 9213 Fernandez-Soto, A., Mannucci, F., Fugazza, D., et al. 2009, GCN Circ., 9222 Krimm, H. A., Beardmore, A. P., Evans, P. A., et al. 2009, GCN Circ., 9198 Oliva, E. 2003, Mem. Soc. Astron. Ital., 74, 118

Olivares, F., Kruehler, T., Greiner, J., \& Filgas, R., et al. 2009, GCN Circ., 9215 Patel, M., Warren, S. J., Mortlock, D. J., \& Fynbo, J. P. U. 2010, A\&A, 512, L3 Salvaterra, R., Della Valle, M., Campana, S., et al. 2009, Nature, 461, 1258 Schlegel, D. J., Finkbeiner, D. P., \& Davis, M. 1998, ApJ, 500, 525 Skrutskie, M. F., Cutri, R. M., Stiening, R., et al. 2006, AJ, 131, 1163 Tanvir, N., Levan, A., Kerr, T., \& Wold, T. 2009a, GCN Circ., 9202 Tanvir, N., Levan, A., Wiersema, K., et al. 2009b, GCN Circ., 9219 Tanvir, N., R., Fox, D. B., Levan, A. J., et al. 2009c, Nature, 461, 1254 Thoene, C. C., D’Avanzo, P., Fernandez-Soto, A., et al. 2009, GCN Circ., 9216 Yoshii, Y., \& Peterson, B. A. 1994, ApJ, 436, 551 